\title{
Guillain-Barré Syndrome Animal Model: The First Proof of Molecular Mimicry in Human Autoimmune Disorder
}

\author{
Nortina Shahrizaila ${ }^{1}$ and Nobuhiro Yuki ${ }^{2}$ \\ ${ }^{1}$ Division of Neurology, Department of Medicine, Faculty of Medicine, University of Malaya, Lembah Pantai, \\ 59100 Kuala Lumpur, Malaysia \\ ${ }^{2}$ Departments of Microbiology and Medicine, National University of Singapore, Science Drive 2, Blk MD4A, Level 5, Singapore 117597
}

Correspondence should be addressed to Nobuhiro Yuki, micyuki@nus.edu.sg

Received 9 September 2010; Accepted 20 October 2010

Academic Editor: Oreste Gualillo

Copyright @ 2011 N. Shahrizaila and N. Yuki. This is an open access article distributed under the Creative Commons Attribution License, which permits unrestricted use, distribution, and reproduction in any medium, provided the original work is properly cited.

Molecular mimicry between self and microbial components has been proposed as the pathogenic mechanism of autoimmune diseases, and this hypothesis is proven in Guillain-Barré syndrome. Guillain-Barré syndrome, the most frequent cause of acute neuromuscular paralysis, sometimes occurs after Campylobacter jejuni enteritis. Gangliosides are predominantly cell-surface glycolipids highly expressed in nervous tissue, whilst lipo-oligosaccharides are major components of the Gram-negative bacterium C. jejuni outer membrane. IgG autoantibodies to GM1 ganglioside were found in the sera from patients with Guillain-Barré syndrome. Molecular mimicry was demonstrated between GM1 and lipo-oligosaccharide of $C$. jejuni isolated from the patients. Disease models by sensitization of rabbits with GM1 and C. jejuni lipo-oligosaccharide were established. Guillain-Barré syndrome provided the first verification that an autoimmune disease is triggered by molecular mimicry. Its disease models are helpful to further understand the molecular pathogenesis as well as to develop new treatments in Guillain-Barré syndrome.

\section{Introduction}

In the 19th century, Robert Koch postulated a causal relationship between a pathogenic microbe and a disease [1]. This was later extended to the role of autoantibodies in the pathogenesis of human disease by Witebsky et al. [2]. In 1957, they proposed the fulfillment of several criteria to proof the pathogenic effects of autoantibodies, namely, the direct demonstration of free, circulating, or cell-bound antibodies by indirect means, the recognition of specific antigen against which the antibody is directed, the production of antibodies against the same antigen in experimental animals and finally the appearance of pathological changes in the corresponding tissues of an actively sensitized experimental model that is similar to that in the human disease.

Taking into account both Koch's and Witebsky's postulates, the term "molecular mimicry" was proposed as a mechanism by which infectious agents trigger an immune response against autoantigens, resulting in the development of autoimmune diseases. Similar criteria must be satisfied to conclude that a disease is triggered by molecular mimicry [3]. They are as follows: (i) the establishment of an epidemiological association between the infectious agent and the immune-mediated disease; (ii) the identification of $\mathrm{T}$ cells or antibodies directed against the patient's target antigens; (iii) the identification of microbial mimics of the target antigen; (iv) reproduction of the disease in an animal model. Although there have been a number of diseases proposed to exhibit the mechanism of molecular mimicry, none has been proven in examples of human diseases based on fulfilment of all four criteria [4].

Guillain-Barré syndrome (GBS), characterized by limb weakness and areflexia, has become the most frequent cause of acute flaccid paralysis since the near elimination of poliomyelitis in the world [5]. Most GBS patients have had either gastrointestinal or upper respiratory symptoms one to three weeks prior to the onset of their neurological symptoms, making GBS the prototype of postinfectious 
autoimmune diseases. GBS can be classified into two major subtypes, acute inflammatory demyelinating polyneuropathy (AIDP) and acute motor axonal neuropathy (AMAN) depending on whether the myelin or the axonal components of the peripheral nerves are affected. Experimental autoimmune neuritis (EAN) resembles AIDP clinically and pathologically. EAN can be transferred to animals by T cells sensitized to peripheral nerve proteins such as P2 protein. However, no investigators have shown conclusive evidence that such autoreactive T-cell response is seen in patients with GBS, indicating that EAN is not a true model of AIDP [6]. In this paper, we describe the development of a true model of AMAN, which fulfills all the four criteria of molecular mimicry as well as Witebsky's postulate as stated above. This verifies GBS as the first paradigm of an autoimmune disease triggered by molecular mimicry. We also discuss how this disease model has helped uncover the precise mechanism of muscle weakness in GBS, which will potentially lead to the development of better treatments.

\section{Proof of Molecular Mimicry Theory}

Gram-negative bacterium Campylobacter jejuni, a leading cause of acute gastroenteritis, is the most common antecedent microorganism in GBS. A prospective casecontrol study detected evidence of recent $C$. jejuni infection in $26 \%$ of patients with GBS in comparison to only $2 \%$ of the household controls (a member of the patient's household) and $1 \%$ of the age-matched hospital controls [7]. That study established an epidemiological association between $C$. jejuni infection and GBS. A study showed that $C$. jejuni infection was associated with AMAN, but not AIDP [8], although this finding has yet to be verified by other investigators.

Autoantibodies are considered to be the pathogenic components which trigger GBS because plasma exchange is proven to be an effective treatment in GBS [9]. Gangliosides constitute a large family of predominantly cell-surface glycosphingolipids bearing a ceramide moiety anchored in the external leaflet of the lipid bilayer and a sialylated oligosaccharide core exposed in the extracellular space. Autopsy studies show that, in AMAN, IgG is deposited on the axolemma of the spinal anterior root [10], indicating that $\operatorname{IgG}$, which binds effectively with complement, is an important factor in the development of AMAN. Patients who develop AMAN subsequent to C. jejuni enteritis have IgG antibodies against GM1 [11]. Their autoantibody titres decrease as the clinical time course progresses. In contrast, patients who have $C$. jejuni enteritis with no neurological sequelae did not have similar autoantibodies. These findings suggest that GM1 is an autoantigen for IgG antibodies in patients with AMAN subsequent to $C$. jejuni enteritis. IgG anti-GDla antibodies are also associated with AMAN subsequent to C. jejuni infection [12].

Lipo-oligosaccharides (LOSs) are a family of phosphorylated glycolipids anchored on the outer surface membrane of C. jejuni. A C. jejuni strain isolated from an AMAN patient carrying IgG anti-GM1 antibody expressed an oligosaccharide structure [Gal $\beta 1-3$ GalNAc $\beta 1-4$ (NeuAc $\alpha 2-3)$ Gal $\beta$ ]
GM1
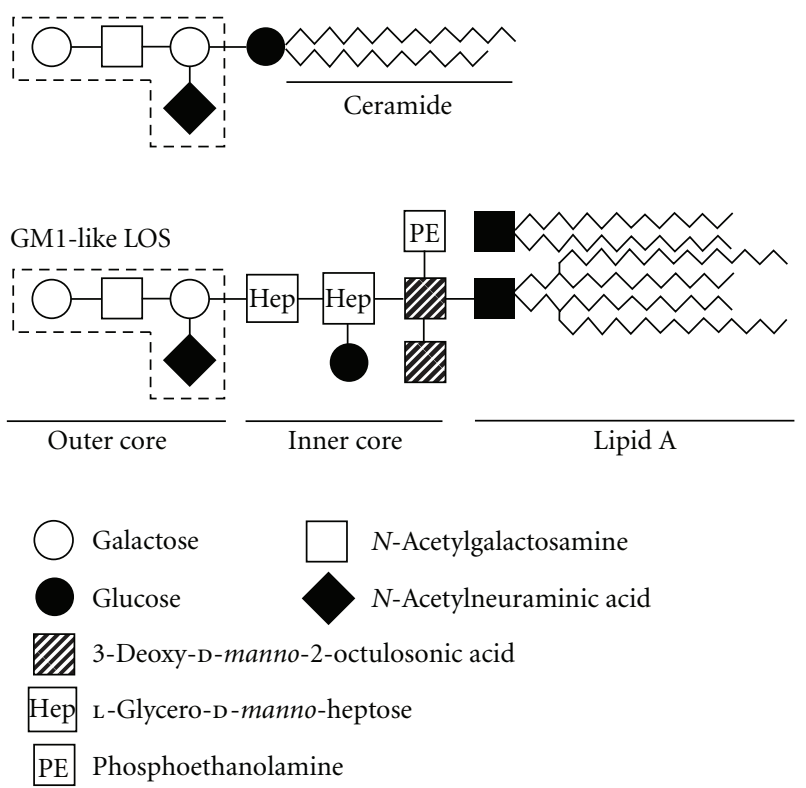

FIGURE 1: Carbohydrate mimicry between gangliosides and Campylobacter jejuni lipo-oligosaccharides (LOSs). The terminal tetrasaccharide of GM1-like LOS is identical to that of GM1 (shown by dash lines).

which protruded from the LOS core (Figure 1) [13]. This terminal structure was identical to that of the terminal tetrasaccharide of the GM1 ganglioside. This was the first definitive evidence of molecular mimicry between human peripheral nerves and antecedent agents in GBS. This strain also carried a GDla-like LOS [14]. Further evidence regarding the role of antiganglioside antibodies in GBS came in the form of adverse reactions to therapeutic gangliosides extracted from bovine brain tissue. These were previously used in the treatment of various neurological disorders. In a study investigating seven patients who developed AMAN following intravenous bovine brain ganglioside administration, anti-GM1 antibodies were discovered in six patients and anti-GD1a and -GT1b antibodies in one patient [15]. These findings set the scene for the work done in the development of a suitable experimental animal model for GBS.

An AMAN model was established by the sensitization of rabbits with a bovine brain ganglioside mixture that included GM1 or with an isolated GM1 [16]. The rabbits developed high titres of IgG anti-GM1 antibodies, followed by an acute onset of flaccid limb weakness with a monophasic course. Pathological findings in their peripheral nerves showed predominant Wallerian-like degeneration with neither lymphocytic infiltration nor demyelination features. IgG was deposited on the axons of the anterior roots, internodal axolemmas, and nodes of Ranvier. Cauda equina and spinal nerve root specimens from the paralyzed rabbits showed macrophage infiltration in the periaxonal space [17]. Surrounding myelin sheaths were almost intact. These findings correspond well with pathological findings for human AMAN $[10,18]$. 


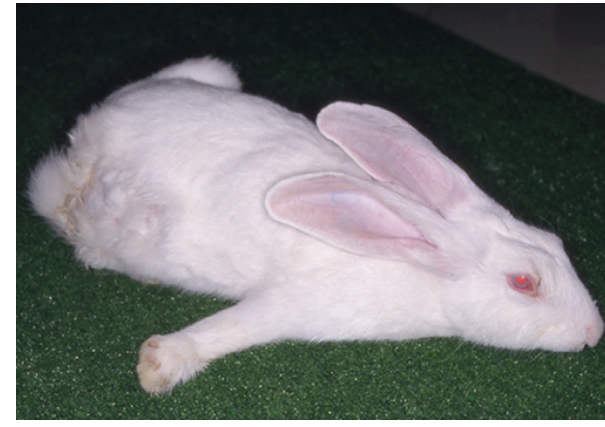

(a)

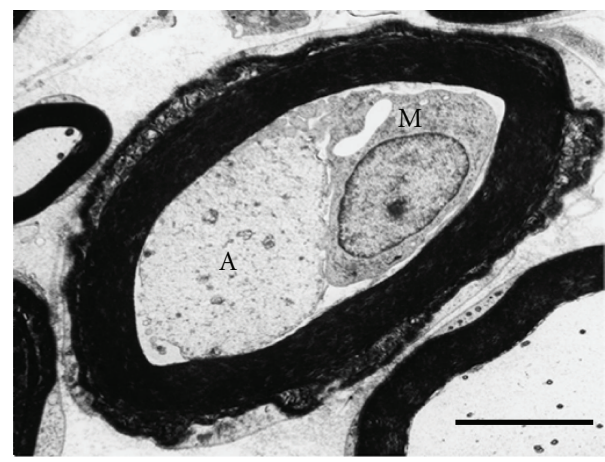

(c)

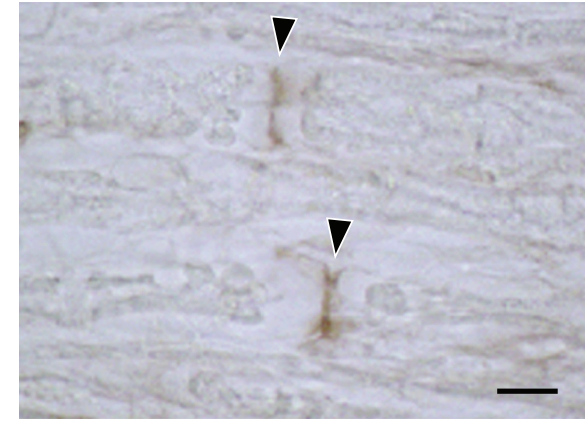

(b)

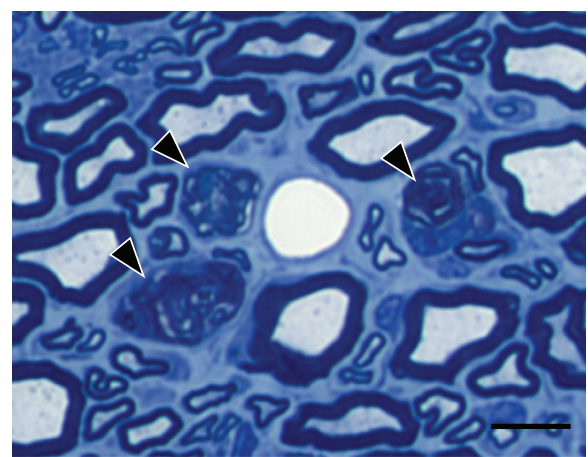

(d)

FIgURE 2: Characteristic findings for the acute motor axonal neuropathy rabbit model. (a) Rabbit with flaccid limb weakness induced by sensitization with Campylobacter jejuni lipo-oligosaccharide. Its body is splayed, all extremities extended, head on the floor not sitting upright in the usual compact, hunched posture. (b) Longitudinal section of the cauda equina. The nodes of Ranvier are stained selectively with protein $\mathrm{G}$ (arrowheads). Scale bar, $10 \mu \mathrm{m}$. (c) Electron micrograph of a nerve fiber with macrophage infiltration. A macrophage (M) occupies the periaxonal space between the atrophic axon (A) and surrounding myelin sheath which appears almost normal. Scale bar, $5 \mu \mathrm{m}$. (d) Wallerian-like degeneration of nerve fibers in a paralyzed rabbit killed 39 days after onset. Sciatic nerve cross section with toluidine blue stain. Myelin ovoids produced by Wallerian-like degeneration of myelinated fibers are present (arrowheads). Scale bar, $10 \mu \mathrm{m}$. Muscle Nerve 35:691-711, Copyright 2007, John Wiley \& Sons, Inc.

The most straightforward way of verifying that molecular mimicry between microbes and autoantigens cause GBS is to establish a GBS model by the immunization of animals with components of antecedent infectious agents. An AMAN model was established by the immunization of rabbits with $C$. jejuni LOS bearing a GM1-like structure [19]. On sensitization with this GM1-like LOS, rabbits developed IgG anti-GM1 antibodies and subsequent flaccid limb weakness (Figure 2). Their nerve roots had occasional macrophages in the periaxonal spaces surrounded by an almost intact myelin sheath. Axons of these nerve fibres showed various degrees of degeneration. Demyelination and remyelination features were rare. These findings, which are compatible with the features of human AMAN, provided the evidence that rabbits inoculated with $C$. jejuni LOS constitute a valid AMAN model. This represents the first replica of human autoimmune disease in an animal model immunized by a microbial mimic of a selfantigen.

In contrast to the active immunization models above, a passive transfer model was developed to further proof the molecular mimicry theory. Ex vivo nerve-muscle preparations from GDla-overexpressing mice were exposed to mouse IgG anti-GD1a monoclonal antibodies in the presence of a complement source [20]. This allowed the morphological and neurophysiological features of such an exposure to be investigated further. Dense antibody and complement deposits were shown to only be present on the presynaptic motor axons. This was accompanied by severe ultrastructural damage and electrophysiological blockade of motor nerve terminal function. A similar paralyzing effect was found when human sera with IgG anti-GD1a reactivity were used instead. The nature of this injury is also complement dependent. These findings indicate that antiGD1a antibodies arise from molecular mimicry and are the likely trigger of peripheral nerve injury.

In summary, GBS is the first true model of molecular mimicry having fulfilled all four criteria as follows: (i) establishment of an epidemiological association between GBS and C. jejuni infection by a prospective case-control study [7], (ii) identification of autoantibodies against GM1 and GDla gangliosides in patients with AMAN subsequent to C. jejuni enteritis $[11,12]$, (iii) identification of molecular mimicry between GM1 or GD1a and LOS of C. jejuni isolated from AMAN [13, 14], and (iv) reproduction of the AMAN models by active immunization of rabbits 
with GM1 or the C. jejuni LOS $[16,19]$ as well as by passive transfer of anti-GD1a antibodies in mice [20]. This makes GBS the first disease in humans to verify that an autoimmune disease is triggered by molecular mimicry.

\section{Development of New Treatments}

In both human and rabbit AMAN, the earliest pathological changes consist of lengthening of the node of Ranvier with distortion of the paranodal myelin [17, 21]. Voltage-gated sodium channel dysfunction is postulated to occur at the nodes of Ranvier [22]. There has been some controversy as to whether anti-GM1 antibodies truly affect sodium channels at the nodes of Ranvier [23, 24]. In the spinal anterior roots of AMAN rabbits, IgG antibodies bind to nodes of Ranvier, where GM1 is highly expressed. This binding of autoantibodies triggers complement activation with deposition of C3 components followed by membrane attack complex formation at the nodal axolemma. The sodium channel clusters are then altered by the destruction of their stabilizing components which include the axonal cytoskeleton at nodes, Schwann cell microvilli, and paranodal axo-glial junctions. This apparent "disappearance" of sodium channel clusters significantly lowers the safety factor for impulse transmission, thus causing muscle weakness in AMAN. This is a novel mechanism by which autoantibodies can modulate sodium channel properties to cause the development of certain neurological disorders.

Plasma exchange and intravenous immunoglobulins are equally effective treatments in GBS [9]. Despite the administration of either immunotherapeutic agent, there are still mortality and significant morbidity in patients with GBS; $3-10 \%$ death and 20\% immobile after 6 months [5]. Research into new treatment options to improve the final outcome of GBS is urgently required. As previously described, complement activation products are deposited on the outer membrane of Schwann cells in AIDP patients [25], and on the axolemma of motor fibres in AMAN [10]. These postmortem studies show that complement activation followed by membrane attack complex formation is an important mechanism for the glial and neuronal injury seen in GBS. This suggests that complement inhibitors have the potential of being a new, more rational treatment for GBS.

Nafamostat mesilate is a synthetic serine protease inhibitor that has been used clinically in Japan for over 20 years with no serious adverse effects to patients. As the complement system contains several serine proteases such as $\mathrm{C} 1 \mathrm{r}, \mathrm{C} 1 \mathrm{~s}$, and $\mathrm{C} 3 / \mathrm{C} 5$ convertases, nafamostat mesilate can efficiently inhibit these serine proteases, thus blocking the formation of the membrane attack complex. This inhibition of complement deposition and sodium channel cluster disappearance has been demonstrated in AMAN rabbits [26]. These experimental findings along with the autopsy findings would justify establishing clinical trials to investigate the use of complement inhibitors as a potential treatment for GBS.

\section{Conclusion}

In this paper, we have demonstrated how the animal model of AMAN has contributed to the proof of the molecular mimicry theory. However, the same cannot be said of AIDP. Only the first criterion has been achieved with the association of AIDP with cytomegalovirus infection. Until the target antigen in AIDP is ascertained, identification of its microbial mimics and reproduction of the disease in an animal model remain unresolved.

\section{Acknowledgment}

The authors thank Akira Hamaoka for preparing Figure 1.

\section{References}

[1] S. Falkow, "Molecular Koch's postulates applied to microbial pathogenicity," Reviews of Infectious Diseases, vol. 10, supplement 2, pp. S274-S276, 1988.

[2] E. Witebsky, N. R. Rose, K. Terplan, J. R. Paine, and R. W. Egan, "Chronic thyroiditis and autoimmunization," JAMA, vol. 164, no. 13, pp. 1439-1447, 1957.

[3] C. W. Ang, B. C. Jacobs, and J. D. Laman, "The GuillainBarré syndrome: a true case of molecular mimicry," Trends in Immunology, vol. 25, no. 2, pp. 61-66, 2004.

[4] N. R. Rose and I. R. Mackay, "Molecular mimicry: a critical look at exemplary instances in human diseases," Cellular and Molecular Life Sciences, vol. 57, no. 4, pp. 542-551, 2000.

[5] P. A. van Doorn, L. Ruts, and B. C. Jacobs, "Clinical features, pathogenesis, and treatment of Guillain-Barré syndrome," The Lancet Neurology, vol. 7, no. 10, pp. 939-950, 2008.

[6] A. K. Asbury and G. M. McKhann, "Changing views of Guillain-Barré syndrome," Annals of Neurology, vol. 41, no. 3, pp. 287-288, 1997.

[7] J. H. Rees, S. E. Soudain, N. A. Gregson, and R. A. C. Hughes, "Campylobacter jejuni infection and Guillain-Barré syndrome," The New England Journal of Medicine, vol. 333, no. 21, pp. 1374-1379, 1995.

[8] S. Kuwabara, K. Ogawara, S. Misawa et al., "Does Campylobacter jejuni infection elicit "demyelinating" Guillain-Barré syndrome," Neurology, vol. 63, no. 3, pp. 529-533, 2004.

[9] R. A. C. Hughes, A. V. Swan, J.-C. Raphaël, D. Annane, R. van Koningsveld, and P. A. van Doorn, "Immunotherapy for Guillain-Barré syndrome: a systematic review," Brain, vol. 130, no. 9, pp. 2245-2257, 2007.

[10] C. Hafer-Macko, S.-T. Hsieh, C. Yan Li et al., "Acute motor axonal neuropathy: an antibody-mediated attack on axolemma," Annals of Neurology, vol. 40, no. 4, pp. 635-644, 1996.

[11] N. Yuki, H. Yoshino, S. Sato, and T. Miyatake, "Acute axonal polyneuropathy associated with anti-GM1 antibodies following Campylobacter enteritis," Neurology, vol. 40, no. 12, pp. 1900-1902, 1990.

[12] T. W. Ho, H. J. Willison, I. Nachamkin et al., "Anti-GD1a antibody is associated with axonal but not demyelinating forms of Guillain-Barré syndrome," Annals of Neurology, vol. 45 , no. 2, pp. 168-173, 1999.

[13] N. Yuki, T. Taki, F. Inagaki et al., "A bacterium lipopolysaccharide that elicits Guillain-Barré syndrome has a GM1 ganglioside-like structure," Journal of Experimental Medicine, vol. 178 , no. 5, pp. 1771-1775, 1993. 
[14] M. Koga, M. Takahashi, M. Masuda, K. Hirata, and N. Yuki, "Campylobacter gene polymorphism as a determinant of clinical features of Guillain-Barré syndrome," Neurology, vol. 65, no. 9, pp. 1376-1381, 2005.

[15] I. Illa, N. Ortiz, E. Gallard, C. Juarez, J. M. Grau, and M. C. Dalakas, "Acute axonal Guillain-Barré syndrome with IgG antibodies against motor axons following parenteral gangliosides," Annals of Neurology, vol. 38, no. 2, pp. 218-224, 1995.

[16] N. Yuki, M. Yamada, M. Koga et al., "Animal model of axonal Guillain-Barré syndrome induced by sensitization with GM1 ganglioside," Annals of Neurology, vol. 49, no. 6, pp. 712-720, 2001.

[17] K. Susuki, M. N. Rasband, K. Tohyama et al., "Anti-GM1 antibodies cause complement-mediated disruption of sodium channel clusters in peripheral motor nerve fibers," Journal of Neuroscience, vol. 27, no. 15, pp. 3956-3967, 2007.

[18] G. M. McKhann, D. R. Cornblath, J. W. Griffin et al., "Acute motor axonal neuropathy: a frequent cause of acute flaccid paralysis in China," Annals of Neurology, vol. 33, no. 4, pp. 333342, 1993.

[19] N. Yuki, K. Susuki, M. Koga et al., "Carbohydrate mimicry between human ganglioside GM1 and Campylobacter jejuni lipooligosaccharide causes Guillain-Barré syndrome," Proceedings of the National Academy of Sciences of the United States of America, vol. 101, no. 31, pp. 11404-11409, 2004.

[20] J. A. Goodfellow, T. Bowes, K. Sheikh et al., "Overexpression of GDla ganglioside sensitizes motor nerve terminals to antiGD1a antibody-mediated injury in a model of acute motor axonal neuropathy," Journal of Neuroscience, vol. 25, no. 7, pp. 1620-1628, 2005.

[21] J. W. Griffin, C. Y. Li, C. Macko et al., "Early nodal changes in the acute motor axonal neuropathy pattern of the GuillainBarré syndrome," Journal of Neurocytology, vol. 25, no. 1, pp. 33-51, 1996.

[22] S. Kuwabara, K. Ogawara, J.-Y. Sung et al., "Differences in membrane properties of axonal and demyelinating GuillainBarré syndromes," Annals of Neurology, vol. 52, no. 2, pp. 180 $187,2002$.

[23] N. Hirota, R. Kaji, H. Bostock et al., "The physiological effect of anti-GM1 antibodies on saltatory conduction and transmembrane currents in single motor axons," Brain, vol. 120, no. 12, pp. 2159-2169, 1997.

[24] T. Takigawa, H. Yasuda, R. Kikkawa, Y. Shigeta, T. Saida, and H. Kitasato, "Antibodies against GM1 ganglioside affect $\mathrm{K}^{+}$ and $\mathrm{Na}^{+}$currents in isolated rat myelinated nerve fibers," Annals of Neurology, vol. 37, no. 4, pp. 436-442, 1995.

[25] C. E. Hafer-Macko, K. A. Sheikh, C. Y. Li et al., "Immune attack on the Schwann cell surface in acute inflammatory demyelinating polyneuropathy," Annals of Neurology, vol. 39, no. 5, pp. 625-635, 1996.

[26] V. Phongsisay, K. Susuki, K. Matsuno et al., "Complement inhibitor prevents disruption of sodium channel clusters in a rabbit model of Guillain-Barré syndrome," Journal of Neuroimmunology, vol. 205, no. 1-2, pp. 101-104, 2008 


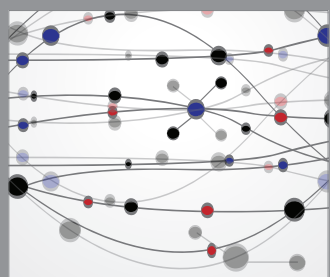

The Scientific World Journal
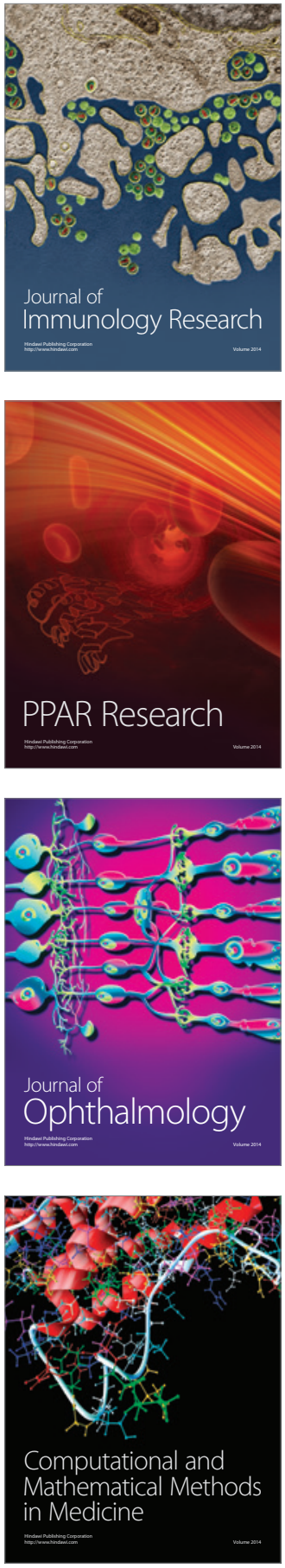

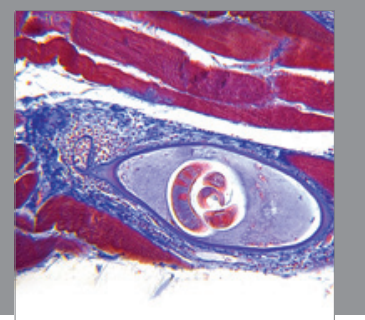

Gastroenterology

Research and Practice
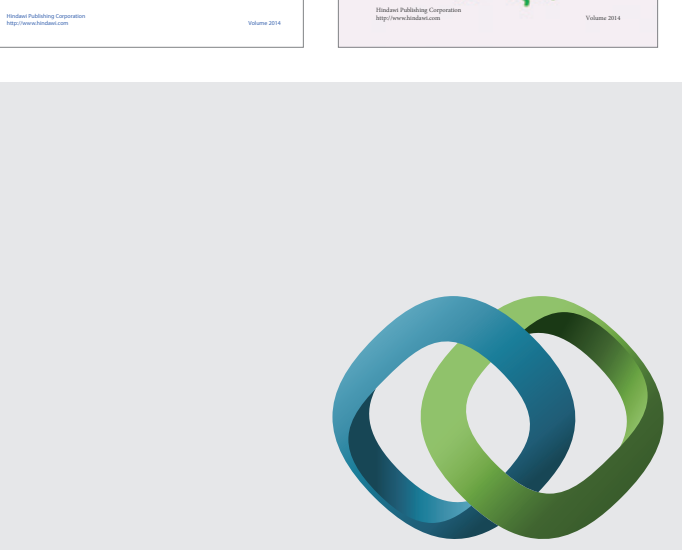

\section{Hindawi}

Submit your manuscripts at

http://www.hindawi.com
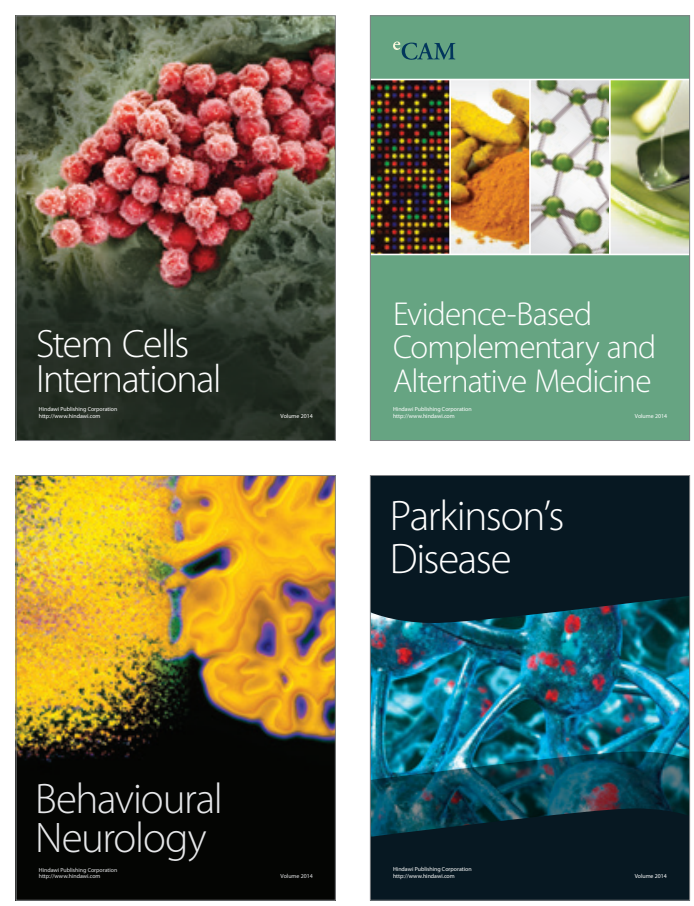

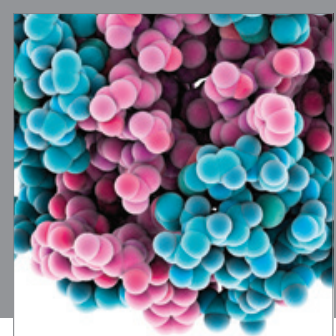

Journal of
Diabetes Research

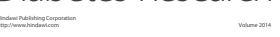

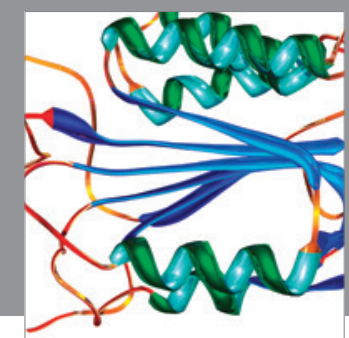

Disease Markers
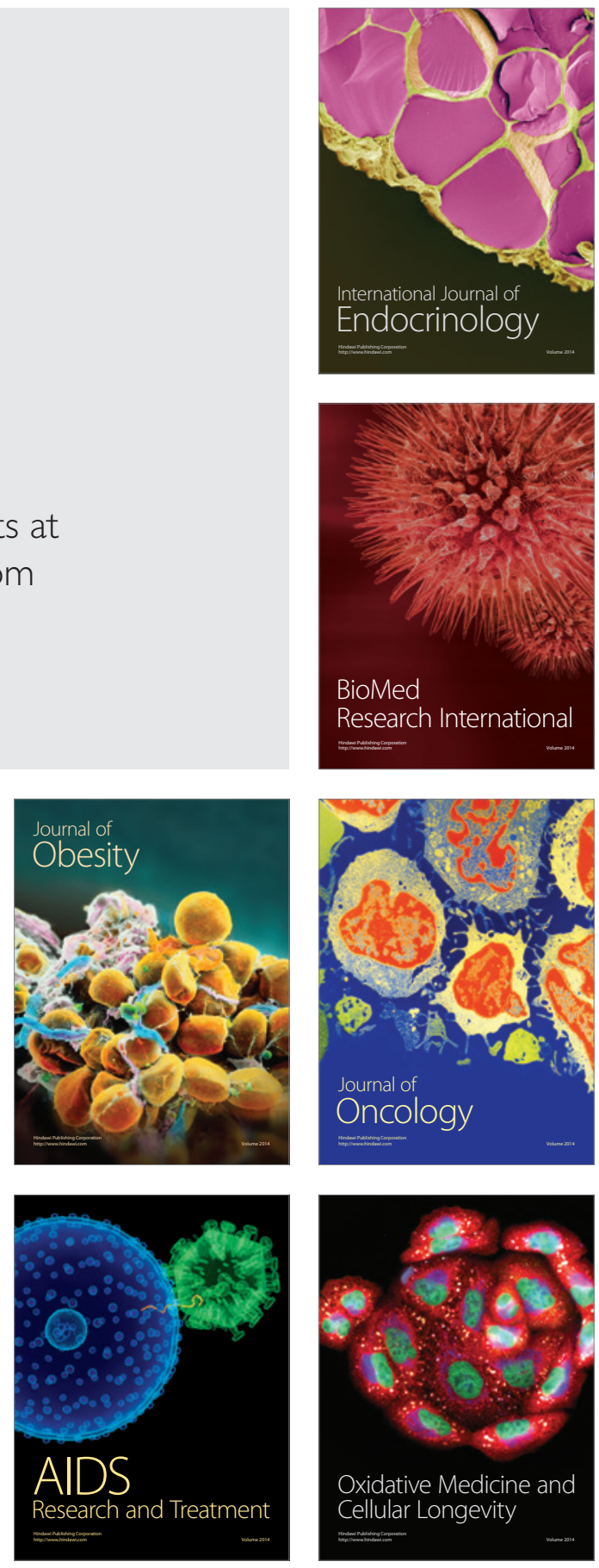